\title{
GESTÃO DE CUSTOS E ENERGIA: EVIDÊNCIAS, OPORTUNIDADES E RESULTADOS OBTIDOS EM UM CASO CONCRETO
}

\author{
Nilmaer Souza da Silva, César Daltóe Berci
}

Universidade do Oeste Paulista - Unoeste. Curso de Administração e Engenharia Elétrica. Presidente Prudente, SP. Email: nilmaer@unoeste.br.

\section{RESUMO}

A aquisição de energia elétrica no Ambiente de Contratação Livre - ACL é uma alternativa consolidada de redução dos custos, visto que possibilita a contratação prévia da energia a ser consumida por um preço livremente negociado entre os players de mercado. O objetivo deste estudo foi demonstrar, por meio de um estudo de caso, como a aquisição de energia elétrica no ACL pode auxiliar na redução dos custos organizacionais e gerar resultados econômicos expressivos. Metodologicamente, tratou-se de pesquisa explicativa, com abordagem quanti-qualitativa, cujos instrumentos de coleta de dados foram a pesquisa bibliográfica e análise documental proveniente de um estudo de caso. A análise dos dados ocorreu por meio de planilhas eletrônicas, gráficos e, por fim, análise de conteúdo e comparativa. Os resultados demonstram a redução de custos de mais de $\mathrm{R} \$$ 5.000,000,00 (cinco milhões de reais), payback inferior a dois meses e uma Taxa Interna de Retorno - TIR de 365\%.

Palavras-chave: Ambiente de Contração Livre, Ambiente de Contratação Regulado, Custos, Energia Elétrica, Economia.

\section{COST AND ENERGY MANAGEMENT: EVIDENCES, OPPORTUNITIES, AND RESULTS OBTAINED IN A CONCRETE CASE}

\begin{abstract}
The acquisition of electricity in the Free Contracting Environment - FCE - is a consolidated alternative of cost reduction, since it allows the previous contracting of the energy to be consumed at a freely negotiated price among market players. The objective of this study was to demonstrate, through a case study, how the acquisition of electric energy in the FCE can help reduce organizational costs and generate significant economic results. Methodologically, it was an explanatory research, with quanti-qualitative approach, whose instruments of data collection were the bibliographical research and documentary analysis coming from a case study. The analysis of the data occurred through electronic spreadsheets, graphics, and, finally, content and comparative analysis. The results demonstrate the cost reduction, of the $\mathrm{R} \$ 5,000,000.00$ (five million "reais"), a lower payback in less than two months, and also an Internal Rate of Return - IRR - of $365 \%$.

Keywords: Unregulated Contracting Environments, Regulated Contracting Environments, Costs, Electricity, Economy.
\end{abstract}

\section{INTRODUÇÃO}

A concorrência, a instabilidade política e econômica que avassalam o Brasil têm impulsionado as organizações a adotarem diversas estratégias de gestão para garantir a permanência no mercado. A estratégia de redução de custos é constantemente aplicada mesmo em cenários favoráveis, visto que possibilita melhorar os resultados da organização, aumentando a margem de lucro e possibilitando o crescimento desta. Os custos são todos os bens, serviços e insumos consumidos na produção de outros bens e serviços (SILVA, 2008; CLEMENTE; SOUZA, 2011). Dentre os custos existentes em uma organização, há os de energia elétrica, que tem um peso significativo na formação de preços tanto dos produtos quanto de serviços prestados pelas organizações. A energia elétrica é um custo 
variável, que sazonaliza-se em função do consumo. (MARTINS, 2010; CLEMENTE; SOUZA, 2011).

No Brasil, o mercado é gerido pelo Ministério de Minas e Energia e regulamentado, notadamente, pela ANEEL - Agência Nacional de Energia Elétrica e dividido em dois grandes ambientes, o Ambiente de Contratação Regulada - ACR e o Ambiente de Contratação Livre - ACL. O ACR, também chamado Mercado Cativo, é a forma tradicional, portanto, mais comum, para se adquirir energia elétrica no Brasil, sendo a única opção dos consumidores residenciais e de algumas empresas, os quais possuem a obrigatoriedade de contratar a energia elétrica da distribuidora de sua região. Já no $A C L, O$ consumidor, atendendo aos pré-requisitos necessários para migração a esse ambiente, pode optar por escolher livremente o seu fornecedor, onde ambos negociarão todas as condições contratuais, incluindo o preço (TORRES, 2006; FLOREZI, 2009; SILVA, 2014; ABRACEEL, 2016; ANEEL, 2016).

O objetivo deste estudo foi demonstrar, por meio de um estudo de caso, como a aquisição de energia elétrica no $\mathrm{ACL}$ pode auxiliar na redução dos custos organizacionais e gerar resultados econômicos expressivos.

\section{METODOLOGIA}

Tratou-se de uma pesquisa explicativa, com abordagem quanti-qualitativa, cujos instrumentos de coleta de dados foram a pesquisa bibliográfica - com extração de informações de períodos, livros, dissertações e documentos públicos - e a análise documental proveniente de um estudo de caso, visto que os dados das instalações foram fornecidos pela empresa pesquisada, objeto do estudo de caso. A análise dos dados ocorreu por meio de planilhas eletrônicas, gráficos e, por fim, análise de conteúdo e análise comparativa, estabelecendo comparações dos resultados entre o ambiente regulado e o livre.

\section{RESULTADOS: O ESTUDO DE CASO.}

O empreendimento objeto de estudo (nome preservado) é um shopping center com mais de duas décadas de atuação, localizado no interior do estado de São Paulo, contando atualmente com 200 operações entre lojas e quiosques de diversos segmentos. Após passar por ampliação no ano de 2013, possui aproximadamente $80.000 \mathrm{~m}^{2}$ de área construída, 1.100 lugares na praça de alimentação e 1.500 vagas de estacionamento. Com cerca de 170 colaboradores próprios, estima-se que, mensalmente, mais de 600 mil pessoas circulam por suas lojas, praça de alimentação e espaços de lazer. Diante destas informações, foi realizado um levantamento do histórico de consumo considerando o período de janeiro de 2010 a julho de 2017, para análise da variação do consumo ao longo do tempo e comparação de custos nos dois ambientes de contratação de energia ( $A C R \times A C L)$. Os dados considerados foram do horizonte janeiro de 2010 a julho de 2017.

\section{Histórico de Consumo de Janeiro de 2010 a Julho de 2017}

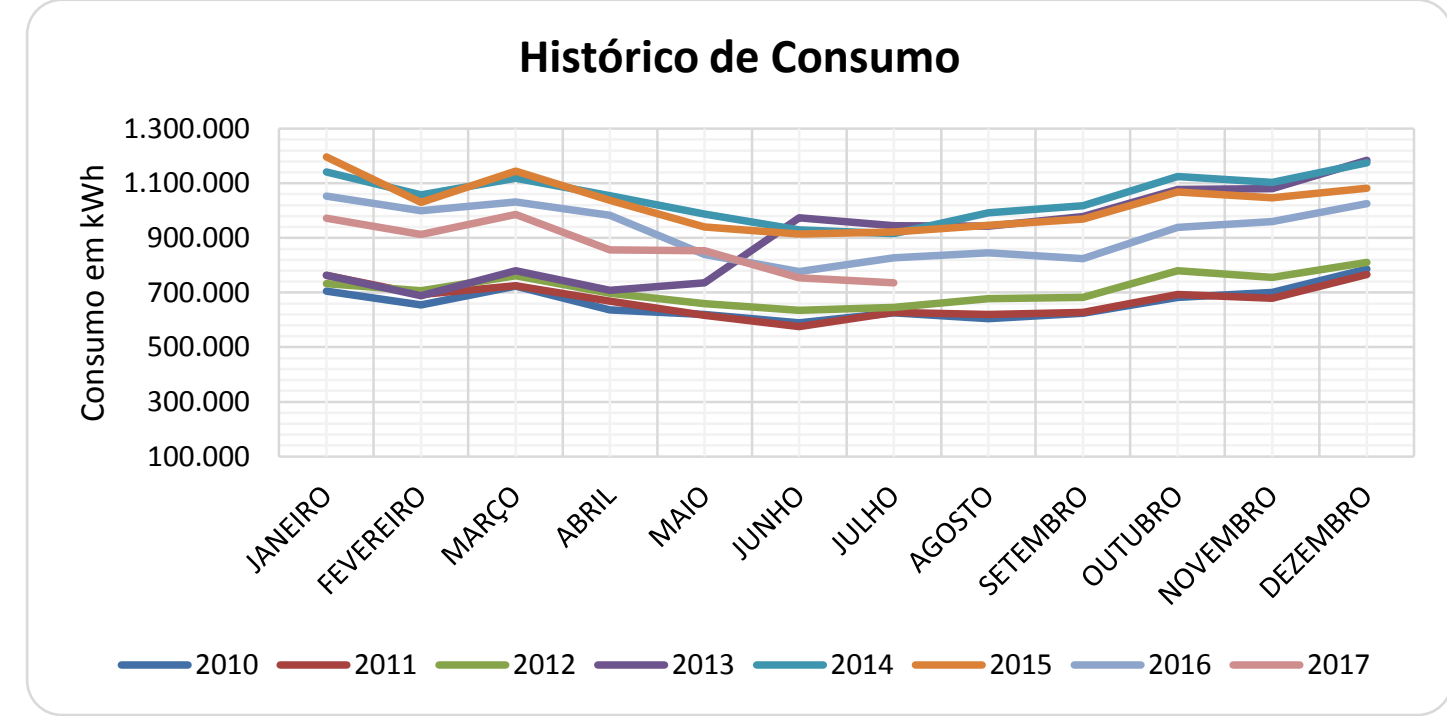

Fonte: Elaborado pelos autores. 
Analisando o gráfico de consumo, foi observado que a partir do mês de junho de 2013 o consumo da unidade elevou-se em virtude da expansão da área, apresentando em média consumos mensais $45 \%$ maiores em relação aos mesmos meses dos anos anteriores. No entanto, de junho de 2014 até o momento, os consumos mensais vêm diminuindo, sendo a causa principal o aumento nos percentuais de vacância, ocasionado pelo cenário econômico apresentado, hoje em torno de $8 \%$ da ABL - Área Bruta Locável. Outro motivo da queda no consumo foi a realização de investimentos em ações que visam eficiência energética, a exemplo da substituição de lâmpadas incandescentes por LED.

\section{DISCUSSÃO}

Como mecanismo de gestão de custos com energia elétrica, utiliza-se, comumente, o valor médio do kWh, que considera, a rigor, todos as grandezas elétricas faturáveis em relação ao consumo total da instalação (SILVA, 2014).

Tabela 1. Custo médio mensal e anual (R\$/kWh).

\begin{tabular}{c|ccccccccccccc}
\hline Ano & Jan & Fev & Mar & Abr & Mai & Jun & Jul & Ago & Set & Out & Nov & Dez & Média \\
\hline $\mathbf{2 0 1 0}$ & 0,32 & 0,35 & 0,31 & 0,33 & 0,37 & 0,37 & 0,38 & 0,37 & 0,37 & 0,37 & 0,41 & 0,37 & 0,36 \\
$\mathbf{2 0 1 1}$ & 0,35 & 0,35 & 0,35 & 0,35 & 0,40 & 0,40 & 0,41 & 0,36 & 0,31 & 0,34 & 0,36 & 0,35 & 0,36 \\
$\mathbf{2 0 1 2}$ & 0,35 & 0,23 & 0,30 & 0,42 & 0,33 & 0,48 & 0,28 & 0,33 & 0,31 & 0,30 & 0,31 & 0,30 & 0,33 \\
$\mathbf{2 0 1 3}$ & 0,31 & 0,27 & 0,26 & 0,30 & 0,30 & 0,30 & 0,27 & 0,26 & 0,27 & 0,27 & 0,28 & 0,27 & 0,28 \\
$\mathbf{2 0 1 4}$ & 0,28 & 0,28 & 0,28 & 0,30 & 0,33 & 0,33 & 0,35 & 0,31 & 0,34 & 0,32 & 0,30 & 0,31 & 0,31 \\
$\mathbf{2 0 1 5}$ & 0,33 & 0,33 & 0,37 & 0,39 & 0,43 & 0,44 & 0,43 & 0,43 & 0,44 & 0,44 & 0,40 & 0,43 & 0,40 \\
$\mathbf{2 0 1 6}$ & 0,46 & 0,45 & 0,43 & 0,44 & 0,48 & 0,50 & 0,47 & 0,52 & 0,55 & 0,54 & 0,51 & 0,50 & 0,49 \\
$\mathbf{2 0 1 7}$ & 0,46 & 0,48 & 0,45 & 0,43 & 0,47 & 0,50 & 0,53 & 0,42 & - & - & - & - & 0,47 \\
\hline Geral & 0,36 & 0,34 & 0,34 & 0,37 & 0,39 & 0,42 & 0,39 & 0,37 & 0,37 & 0,37 & 0,37 & 0,36 & 0,38 \\
\hline
\end{tabular}

Fonte: Elaborado pelos autores.

Considerando que a migração do consumidor para o $\mathrm{ACL}$ ocorreu em agosto de 2011, observa-se quedas anuais no custo médio aplicado por kWh em torno de 9 a $22 \%$ até o ano de 2014, sendo que o custo médio ficou em R\$ 0,31/kWh. Nos anos de 2015 e 2016, o custo médio em $\mathrm{R} \$ / \mathrm{kWh}$ foi de $\mathrm{R} \$ 0,40 / \mathrm{kWh}$ e $\mathrm{R} \$ 0,49 / \mathrm{kWh}$, respectivamente, destacando-se que em 2016, o primeiro semestre apresentou uma tarifa média de $\mathrm{R} \$ 0,46 / \mathrm{kWh}$, e o segundo semestre de $\mathrm{R} \$ 0,52 / \mathrm{kWh}$. Este aumento de 30\% em comparação as médias de 2015 e 2014 foi ocasionado, principalmente, pela aplicação de dois reajustes (extraordinário e periódico) na fatura da distribuidora. Já em 2016, a diferença é justificada pelo início de um novo contrato de comercialização no mês de agosto. Em contrapartida, analisando os dados coletados até o faturamento do consumo de Julho de 2017, o custo médio foi de $\mathrm{R} \$ 0,47 / \mathrm{kWh}$, devido a diminuição do preço da energia, já prevista em contrato de aquisição e a venda de sobras contratuais nos meses de janeiro, fevereiro, abril, junho e julho, além da solicitação de redução do MUSD - Montante de Uso do Sistema de Distribuição contratado com a distribuidora, todos estes, itens de gerenciamento de custos energéticos.

Ainda sobre os resultados da migração deste consumidor, caso seja comparada a tarifa média do mês de migração (agosto de 2011) com a de agosto de 2017, tem-se uma elevação de $16 \%$. Nesse mesmo interstício, a inflação acumulada pelo ${ }^{1}$ IGPM-FGV foi de $38,90 \%$ (BANCO CENTRAL DO BRASIL, 2017). Caso estivesse no mercado cativo, neste mês, o custo médio da tarifa de energia elétrica seria de $\mathrm{R} \$ 0,61 / \mathrm{kWh}$. 
Tabela 2. Economia auferida (ACR $\times A C L)$

\begin{tabular}{|c|c|c|c|c|}
\hline \multicolumn{5}{|c|}{ RESULTADO DO PROCESSO DE MIGRAÇÃO DO ACR PARA O ACL } \\
\hline ITEM & 2011 & 2012 & 2013 & 2014 \\
\hline ACR & $\mathrm{R} \$ 1.388 .136,33$ & $\mathrm{R} \$ 3.418 .731,85$ & $\mathrm{R} \$ 3.801 .731,95$ & $R \$ 4.511 .947,96$ \\
\hline ACL & $\mathrm{R} \$ 1.173 .759,89$ & $\mathrm{R} \$ 2.944 .291,37$ & $R \$ 3.093 .951,81$ & $R \$ 3.723 .922,24$ \\
\hline (\%) REDUÇÃO & $-15,44 \%$ & $-13,88 \%$ & $-18,62 \%$ & $-17,47 \%$ \\
\hline Economia (R\$) & $\mathrm{R} \$ \mathbf{2 1 4 . 3 7 6 , 4 4}$ & $\mathrm{R} \$ \mathbf{4 7 4 . 4 4 0 , 4 8}$ & $\mathrm{R} \$ \mathbf{7 0 7 . 7 8 0 , 1 4}$ & $\mathrm{R} \$ \mathbf{7 8 8 . 0 2 5 , 7 2}$ \\
\hline ITEM & 2015 & 2016 & 2017 & TOTAL \\
\hline ACR & $\mathrm{R} \$ 6.717 .392,57$ & $R \$ 6.254 .960,71$ & $R \$ 3.528 .900,61$ & $R \$ 29.621 .801,98$ \\
\hline ACL & $\mathrm{R} \$ 5.030 .104,94$ & $R \$ 5.500 .691,29$ & $\mathrm{R} \$ 2.882 .633,30$ & $\mathrm{R} \$ 24.349 .354,84$ \\
\hline \% REDUÇÃO & $-25,12 \%$ & $-12,06 \%$ & $-18,31 \%$ & $-17,80 \%$ \\
\hline Economia (R\$) & $\mathrm{R} \$ \mathbf{1 . 6 8 7 . 2 8 7 , 6 3}$ & $\mathrm{R} \$ \mathbf{7 5 4 . 2 6 9 , 4 2}$ & $\mathrm{R} \$ \mathbf{6 4 6 . 2 6 7 , 3 1}$ & $\mathrm{R} \$ 5.272 .447,14$ \\
\hline
\end{tabular}

Com base na tabela acima, foi observado que em todos os anos desde a migração foi possível auferir economia. Especificamente $o$ ano de 2015, apresentou percentual notavelmente maior de economia devido ao cenário energético ter apresentado redução da oferta de energia, proveniente, sobretudo, das condições hidrológicas desfavoráveis, fator que afetou diretamente os consumidores cativos, inclusive com aplicação de bandeira tarifária vermelha nos três últimos trimestres.

Já no ano de 2016, a proximidade dos custos nos ambientes livre e cativo é maior, devido ao novo contrato de aquisição de energia elétrica vigente a partir do mês de agosto com aplicação de preço $69 \%$ maior que o PLD médio vigente nos últimos meses do ano, em um cenário com sobras contratuais de energia e aplicação de bandeira tarifária verde em grande parte do ano. De forma geral, decorridos seis anos da migração do ambiente cativo para o ambiente livre, a economia auferida pelo consumidor foi de $R \$ 5.272 .447,14$, o equivalente ao valor total pago em 12 meses de consumo e em outra análise, uma economia de $17,8 \%$ do valor que seria pago no ambiente de contratação regulado.

\section{Payback do Investimento}

Segundo Gitman (2010, p.366), “[...] O período de payback é o tempo necessário para que a empresa recupere o investimento inicial em um projeto, calculado a partir das entradas de caixa". Desta forma, considerando um investimento inicial de $\mathrm{R} \$ \mathbf{7 7 . 7 4 0 , 0 0}$ no sistema de medição e faturamento exigido para a migração, o payback do investimento, ou seja, o tempo total de retorno do investimento à empresa foi de um mês e 25 dias, dada a economia de $\mathrm{R} \$ 43.572,55$ no primeiro mês de faturamento e $\mathrm{R} \$ 41.894,83$ no segundo mês.

\section{Valor Presente Líquido - VPL}

Conforme Camloffski (2014), o cálculo do valor presente líquido visa demonstrar o ganho financeiro previsto para o projeto em valores atuais, descapitalizando todos os valores do fluxo de caixa, diminuindo o resultado pelo investimento inicial. 
Tabela 3. Determinação do Valor Presente Líquido (VPL)

CUSTO DE CAPITAL

$10 \%$

INVESTIMENTO

$-R \$ 77.740,00$

\begin{tabular}{c|c}
\hline ANO & ECONOMIA \\
\hline 2011 & $\mathrm{R} \$ 214.376,44$ \\
2012 & $\mathrm{R} \$ 474.440,48$ \\
2013 & $\mathrm{R} \$ 707.780,14$ \\
2014 & $\mathrm{R} \$ 788.025,72$ \\
2015 & $\mathrm{R} \$ 1.687 .287,63$ \\
2016 & $\mathrm{R} \$ 754.269,42$ \\
2017 & $\mathrm{R} \$ 646.267,31$ \\
\hline VPL & $\mathrm{R} \$ \mathbf{3 . 3 8 4 . 3 2 0 , 7 2}$ \\
\hline
\end{tabular}

Fonte: Elaborado pelos autores.

Considerando uma economia auferida de $\mathrm{R} \$$ 5.272.447,14, um custo de capital de $10 \%$ e um investimento inicial de $\mathrm{R} \$ 77.740,00$, o VPL, ou seja, o ganho financeiro para a empresa devido à migração para o $A C L$ foi de $R \$$ 3.384.320,72.

\section{Taxa Interna de Retorno - TIR}

A Taxa Interna de Retorno, de acordo com Gitman (2010, p.371), "é a taxa de retorno anual composta que a empresa obterá, se investir no projeto e receber as entradas de caixa previstas". Tendo em vista o investimento inicial e as economias auferidas, obteve-se uma TIR de $365 \%$, maior do que a taxa de custo de capital de $10 \%$ considerado na determinação do VPL, demonstrando total viabilidade/retorno do projeto.

\section{CONCLUSÃO}

Em um cenário econômico e politicamente instável, as empresas têm encontrado um meio de garantirem sua estabilidade no mercado, aplicando a estratégia de redução de custos. Comumente utilizada em qualquer cenário, essa estratégia pode ser aplicada de diversas formas, como na gestão do insumo energia elétrica que atualmente é comercializada em dois grandes mercados no Brasil: ACR e ACL. Verificou-se que a contratação da energia elétrica no $A C L$ (mercado livre) é viável àqueles consumidores com elevado consumo, a exemplo da empresa objeto de estudo que, em seis anos, auferiu uma economia de mais de cinco milhões, com ganhos reais superiores a três milhões de reais, com uma TIR de $365 \%$ e payback inferior a dois meses.
Desta forma, este estudo beneficiou aos gestores e futuros gestores de grandes organizações demonstrando um método de redução significativa dos custos, maximizando seus resultados sem gerar impactos operacionais, bem como beneficiou a população acadêmica de um modo geral, em virtude principalmente de tratar de um assunto relativamente recente $e$ pouco explorado pela comunidade acadêmica. Recomenda, sobretudo, a contração de consultorias e assessorias disponíveis no mercado para essa finalidade, dada a complexidade regulatória e normativa do setor elétrico, bem como a difusão de casos de sucesso como este, para que haja encorajamento da adoção desta medida por outras organizações empresariais.

\section{REFERÊNCIAS}

ABRACEEL. Cartilha Mercado Livre de Energia Elétrica: Um guia básico para consumidores potencialmente livres e especiais. Brasília, 2016.

ANEEL. Informações Gerenciais. 2016. Disponível em:

$<$ http://www.aneel.gov.br/documents/656877/1 4854008/Boletim+de+Informa\%C3\%A7\%C3\%B5e s+Gerenciais+4\%C2\%BA+trimestre+de+2016/2cc 14375-3e1c-9dfe-f6a6-a5a1fd69f021>. Acesso em: 31 maio 2017.

BANCO CENTRAL DO BRASIL. Histórico das taxas de juros. 2017. Disponível em: <https://www.bcb.gov.br/Pec/Copom/Port/taxaS elic.asp>. Acesso em: 24 mai. 2017.

CAMLOFFSKI, R. Análise de investimentos e viabilidade financeira das empresas. 1. ed. São Paulo: Atlas, 2014. 
CLEMENTE, A.; SOUZA, A. Gestão de custos: aplicações operacionais e estratégicas: exercícios resolvidos e propostos com utilização do Excel. 2. ed. São Paulo: Atlas, 2011.

FLOREZI, G. Consumidores livres de energia elétrica: uma visão prática. 2009, 158f. Trabalho de Conclusão de Curso (Mestrado em Engenharia Elétrica) - Universidade de São Paulo, São Paulo, 2009.

GITMAN, L. J. Princípios de Administração Financeira. 12. ed. São Paulo: Pearson Prentice Hall, 2010.

MARTINS, E. Contabilidade de custos. 10. ed. São Paulo: Atlas, 2010.

SILVA, L. S. Apuração dos custos no principal produto oferecido por uma empresa do ramo alimentício baseado no custeio variável. 2008. 58 f. Trabalho de Conclusão de Curso (Graduação em Ciências Contábeis) - Universidade Federal de Santa Catarina, Florianópolis, 2008.

SILVA, N. S. Análise das emissões de dióxido de carbono e impacto econômico da substituição do diesel por biodiesel na geração termelétrica. 2014. 88f. Dissertação (Mestrado) - Universidade Estadual de Londrina, Londrina, 2014.

TORRES, R. C. Avaliação de portfolios de contratos de compra e venda de energia elétrica: Uma abordagem pela teoria de opções. 2006. 117f. Dissertação (Mestrado) - Pontifícia Universidade Católica do Rio de Janeiro, Rio de Janeiro, 2006.

Recebido para publicação em 13/05/2018

Revisado em 31/07/2018

Aceito em 03/08/2018 